\title{
MGF-based Analysis of Maximum Ratio Combining Receiver over Fisher-Snedecor Composite Fading Channel
}

\author{
Hari Shankar, Ankush Kansal
}

\begin{abstract}
Fisher Snedecor composite fading model is the combination of Nakagami-m and inverse Nakagami-m distribution. The Nakagami-m is used to characterize the small scale fading, whereas shadowing is modeled by inverse Nakagami-m distribution. In this paper, the closed-form expression for moment generating function (MGF) of instantaneous signal to noise ratio (SNR) over independent identically distributed (i.i.d) Fisher-Snedecor composite fading channel using maximum ratio combining (MRC) diversity technique is derived. By using newly derived MGF expression, we derive the closed-form expressions of average bit error rate $(A B E R)$ or average symbol error rate (ASER) for different binary and multilevel modulation schemes. The expressions for average channel capacity (ACC) under two adaptive transmission protocols like optimum rate adaption (ORA) and channel inversion with fixed rate (CIFR) are also derived using proposed MGF. Further, the numerical results of newly derived expression are presented and compared with the results of Rayleigh and Nakagami-m distribution which is the special case of Fisher Snedecor composite fading model.
\end{abstract}

Index Terms: Fading, shadowing, maximum ratio combining, channel capacity, error probability.

\section{INTRODUCTION}

The diversity combining techniques have been extensively used to combat the fading effects that improve the performance of wireless communication systems. In this technique, two or more copies of the same information-bearing signals are combined in order to improve the overall SNR. There are mainly four types of combining techniques: Maximum ratio combining (MRC), equal gain combining (EGC), selection combining (SC) and switch combining (SWC) [1]. Out of them, the MRC technique is an optimal and most commonly used method over any given fading channels. In the previous literature, lots of works have been done using MRC reception algorithm over different fading channels $[1,2]$.

The statistical parameters such as probability density function (PDF), cumulative distribution function (CDF) and MGF are the most important tools for obtaining the performances of digital communication systems. These performance matrices include outage probability, amount of fading, ACC and ASER.

Revised Manuscript Received on July 22, 2019.

Hari Shankar, Ankush Kansal, ECED, Thapar Institute of Engineering and Technology, Patiala, Punjab, India.
The performance analysis using PDF-based approach is analytically difficult and generally, the solution is not presented in the closed form. On the other hand, the MGF based approach provides the closed form solution (in most cases there is no need to solve integration). Therefore, MGF based performance analysis is the most widely accepted technique in wireless communication systems. Unified MGF based approach was presented in [2-4] that has been used to obtain the error probability over various fading channel. The MGF based exact channel capacity analysis was present in [5]. After that, an alternative MGF based exact channel capacity analysis was given in [6].

Composite fading model has an important role to characterize the behavior of the received signal in a real wireless environment. Various composite fading models have been proposed in previous literature [1, 2]. Lognormal distribution based composite fading models and their performance analysis was analytically complex. Generally, their solution was not obtained in closed form, therefore researchers move towards gamma distribution based composite fading model. The examples of gamma distribution based composite fading model are $\mathrm{K}$ distribution [7],

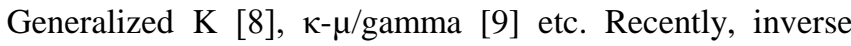
Nakagami-m distribution based composite fading model, known as Fisher-Snedecor, has been proposed [10]. It is the combination of Nakagami-m and inverse Nakagami-m distribution [10]. The Nakagami-m is used to characterize the small scale fading whereas shadowing is model by inverse Nakagami-m distribution. This composite fading model is presented in an algebraic form which is more simple and compliant. In [10], the channel measurement results for device to device communication systems showed good agreement with Fisher fading model as compared to Generalized-K fading model. This composite fading model has two parameters: fading severity $(\mathrm{m})$ and shadowing parameter $\left(\mathrm{m}_{\mathrm{s}}\right)$.

The work on Fisher fading model has been reported in various literature [11-18]. In [10], the expressions for PDF, CDF, MGF, outage probability, amount of fading and ABER for binary phase shift keying (BPSK) and differential phase shift keying (DPSK) over Fisher fading channel were derived. The exact closed form and asymptotic expressions of SER for $\mathrm{M}$-ary pulse amplitude modulation (M-PAM) and M-ary

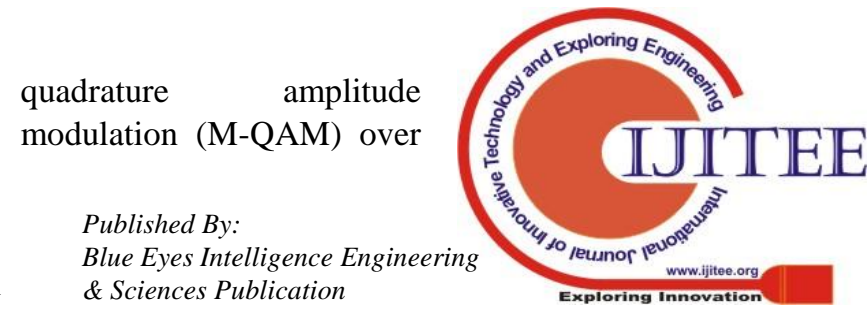


Fisher fading channel were derived in [11]. In [11], the author further derived the exact closed-form expression for ACC. The closed-form expressions for average probability of detection (PD), average area under receiver operating characteristic (AUC) and effective rate over Fisher-Snedecor and $\kappa-\mu$ shadowed fading channel were derived in [12]. The closed form expression for generalized MGF of Fisher fading model was derived in [13]. By using generalized MGF expression, the author, in [13], further derived the expression for the probability of successful secure transmission. The closed form expressions of effective rates for multiple input single output (MISO) system over both independent but not identically (i.i.n.d) and independent identically distributed (i.i.d) Fisher fading channel were given in [14]. The exact closed-form expressions for the probability of non-zero secrecy capacity, secrecy outage probability and average secrecy capacity over Fisher fading channel were derived. In [16], the closed form expression for effective capacity over Fisher fading channel was derived. In [17], the channel capacity under different adaptive transmission policies such as ORA, optimal power and rate adaption (OPRA), CIFR and truncated CIFR over Fisher fading channel was derived. In [18], the expression for MGF of instantaneous SNR over Fisher fading channel was derived. By using derived MGF, the author further obtained the expressions for PDF and CDF of the sum of independent but not identically (i.n.i.d) distributed Fisher- Snedecor variates, which was employed in the analysis of MRC receiver [18]. The author further derived the expressions for outage probability, outage capacity and ABER for BPSK modulation schemes [18].

In this paper, we derive a new closed-form expression for MGF of instantaneous SNR over independent identically distributed (i.i.d) MRC receiver of Fisher fading model. By using derived MGF expression, we derive the closed-form expressions of ABER for binary and multilevel modulation schemes. Moreover, the expressions for average channel capacity for ORA and CIFR policy are also obtained using derived MGF expression. The obtained expressions are presented in the term of Mac Robert, Meijer-G and hypergeometric function. Further, the numerical results are presented by using the newly derived expression. Moreover, some results are also compared with the results of Rayleigh and Nakagami-m distribution.

The rest of paper is organized as follows. In Section II, the PDF of Fisher fading model with MRC receiver is presented. The expression for MGF using i.i.d MRC algorithm is derived in Section III. The expressions of ABER for different modulation schemes and ACC with MRC receiver are derived in Section IV and Section V, respectively. Section VI presents the numerical results of evaluated performance matrices. The work of the paper is summarized with the conclusion in Section VII.

\section{FISHER SNEDECOR COMPOSITE FADING CHANNEL}

The Fisher Snedecor composite fading model is the mixture of Nakagami-m and inverse Nakagami-m distribution. The PDF of this composite fading model is defined by [10],

$$
f_{\gamma}(\gamma)=\frac{m^{m} \cdot\left(m_{s} \bar{\gamma}\right)^{m_{s}} \gamma^{m-1}}{B\left(m, m_{s}\right)\left(m \gamma+m_{s} \bar{\gamma}\right)^{m+m_{s}}}
$$

where, $m$ and $m_{s}$, represents the fading severity and shadowing parameter, respectively. $\gamma$ is the instantaneous SNR of the received signal. $\bar{\gamma}=E[\gamma]$ is the average SNR. $\mathrm{B}(\cdot, \cdot)$ is the beta function [19]. This composite fading model comprises two special cases such as Rayleigh and Nakagami-m distribution. By substituting, $\mathrm{m}=1$ under light shadowing $\left(\mathrm{m}_{\mathrm{s}} \rightarrow \infty\right)$, it reduces to Rayleigh distribution. With $\mathrm{m}_{\mathrm{s}} \rightarrow \infty$, it reduces to Nakagami-m distribution having the same Nakagami-m parameter as fading severity parameter (m) [10].

Let us consider L independent identically distributed (i.i.d) branch MRC diversity receiver operating over Fisher fading channel. The PDF of instantaneous SNR with MRC receptor is given by [18],

$$
\begin{gathered}
f_{\gamma}(\gamma)_{M R C}=\frac{1}{\Gamma(m L)}\left(\frac{m}{m_{s} \bar{\gamma}}\right)^{m L}\left(\frac{\Gamma\left(m+m_{s}\right)}{\Gamma\left(m_{s}\right)}\right)^{L} \\
\quad \times \gamma^{m L-1}{ }_{2} F_{1}\left(m+m_{s}, m L ; m L, \frac{-m \gamma}{m_{s} \bar{\gamma}}\right)
\end{gathered}
$$

where, $\mathrm{L}$ is the number of branches at receiver section. ${ }_{2} \mathrm{~F}_{1}$ is the hypergeometric function [19] and $\Gamma(\cdot)$ is the gamma function [19].

\section{Moment Generating FunCtion}

The MGF is the most important statistical parameter to evaluate the performances of the digital communication system. By using MGF expression, we can elaborate the error rate, outage probability, moment and channel capacity. The MGF of instantaneous SNR is defined by [2],

$$
M_{M R C}(s)=\int_{0}^{\infty} \exp (-s \gamma) f_{M R C}(\gamma) d \gamma
$$

where, $f_{M R C}(\gamma)$ is the PDF of instantaneous SNR over i.i.d MRC fading channel. By substituting (2) into (3), we get,

$$
\begin{gathered}
M_{M R C}(s)=\frac{1}{\Gamma(m L)}\left(\frac{m}{m_{s} \bar{\gamma}}\right)^{m L}\left(\frac{\Gamma\left(m+m_{s}\right)}{\Gamma\left(m_{s}\right)}\right)^{L} \\
\times \int_{0}^{\infty} \exp (-s \gamma) \gamma_{2}^{m L-1} F_{1}\left(m+m_{s}, m L ; m L, \frac{-m \gamma}{m_{s} \bar{\gamma}}\right) d \gamma
\end{gathered}
$$

With the help of table of integral series and product [19, eq.(7522.1)], the above equation can be written as,

$$
\begin{aligned}
M_{M R C}(s) & =\frac{1}{\Gamma(m L) \Gamma\left(m+m_{s}\right)}\left(\frac{m}{m_{s} \bar{\gamma}}\right)^{m L}\left(\frac{\Gamma\left(m+m_{s}\right)}{\Gamma\left(m_{s}\right)}\right)^{L} \\
& \times s^{-m L} E\left(m+m_{s}, m L, m L: m L: s\right)
\end{aligned}
$$

where, $\mathrm{E}(\cdot, \cdot, \because \because \because \cdot)$ is the Mac Robert function [19]. This is the closed form expression of MGF over Fisher fading channel using MRC reception algorithm. An alternative expression of MGF can also be obtained by converting 
hypergeometric function, presented in (4), into Meijer G function [19],

$$
\begin{aligned}
{ }_{2} F_{1}\left(m+m_{s}, m L ; m L, \frac{-m \gamma}{m_{s} \bar{\gamma}}\right) \\
=\frac{m \gamma}{\Gamma\left(m+m_{s}\right) m_{s} \bar{\gamma}} G_{2,2}^{1,2}\left(\frac{m \gamma}{m_{s} \bar{\gamma}} \mid \begin{array}{cc}
-m-m_{s} & -m L \\
-1 & -m L
\end{array}\right)
\end{aligned}
$$

By plugging (6) into (4), we get,

$$
\begin{gathered}
M_{M R C}(s)=\frac{1}{\Gamma(m L) \Gamma\left(m+m_{s}\right)}\left(\frac{m}{m_{s} \bar{\gamma}}\right)^{m L+1}\left(\frac{\Gamma\left(m+m_{s}\right)}{\Gamma\left(m_{s}\right)}\right)^{L} \\
\quad \times \int_{0}^{\infty} \gamma^{m L} \exp (-s \gamma) G_{2,2}^{1,2}\left(\frac{m \gamma}{m_{s} \bar{\gamma}} \mid \begin{array}{cc}
-m-m_{s} & -m L \\
-1 & -m L
\end{array}\right)
\end{gathered}
$$

By using the table of integral series and product [19, eq.7.831.1] and after some mathematical calculation, we get,

$$
\begin{aligned}
& M_{M R C}(s)=\frac{1}{\Gamma(m L) \Gamma\left(m+m_{s}\right)}\left(\frac{m}{s m_{s} \bar{\gamma}}\right)^{m L+1} \\
& \times\left(\frac{\Gamma\left(m+m_{s}\right)}{\Gamma\left(m_{s}\right)}\right)^{L} G_{3,2}^{1,3}\left(\frac{m}{s m_{s} \bar{\gamma}} \mid \begin{array}{ccc}
-m L & -m-m_{s} & -m L \\
-1 & -m L
\end{array}\right)
\end{aligned}
$$

This is an alternate closed-form expression for MGF of instantaneous SNR of i.i.d MRC receiver.

\section{BIT ERROR RATE ANALYSIS}

The SER/BER is an important performance metric to design the digital communication system. The error rate performance over independent identically distributed (i.i.d) fading channel using the MRC algorithm depends on the number of branches (L), fading parameter and SNR output. The average BER/SER over any fading channel can be defined by [2],

$$
P_{e}(E)=\int_{0}^{\infty} P_{e}(E / \gamma) f_{M R C}(\gamma) d \gamma
$$

\section{A. BPSK, BFSK and BFSK with minimum correlation}

The ABER for binary phase shift keying (BPSK) and binary frequency shift keying (BFSK) is given by [20],

$$
P_{e}(E)=\frac{1}{\pi} \int_{0}^{\pi / 2} M_{M R C}\left(\frac{b}{\sin ^{2} \theta}\right) d \theta
$$

where, $b=1$ for BPSK, $b=0.5$ for BFSK and $b=0.715$ for BFSK with minimum correlation. Now, by substituting (8) into (10), we get,

$$
\begin{aligned}
& P_{e}(E)=\frac{1}{\pi \Gamma(m L) \Gamma\left(m+m_{s}\right)}\left(\frac{\Gamma\left(m+m_{s}\right)}{\Gamma\left(m_{s}\right)}\right)^{L}\left(\frac{m}{m_{s} \bar{\gamma}}\right)^{m L+1} \\
& \times \int_{0}^{\pi / 2}\left(\frac{\sin ^{2} \theta}{b}\right)^{m L+1} G_{3,2}^{1,3}\left(\frac{m \sin ^{2} \theta}{b m_{s} \bar{\gamma}} \mid \begin{array}{ccc}
-m L & -m-m_{s} & -m L \\
-1 & -m L
\end{array}\right) d \theta
\end{aligned}
$$

Let $\sin ^{2} \theta=t$ i.e. $2 \sin \theta \cos \theta d \theta=d t$, after some algebraic calculation, (11) can be written as,

$$
P_{e}(E)=\frac{1}{2 \pi \Gamma(m L) \Gamma\left(m+m_{s}\right)}\left(\frac{\Gamma\left(m+m_{s}\right)}{\Gamma\left(m_{s}\right)}\right)^{L}\left(\frac{m}{b m_{s} \bar{\gamma}}\right)^{m L+1}
$$

$$
\times \int_{0}^{1} t^{m L+1 / 2}(1-t)^{-1 / 2} G_{3,2}^{1,3}\left(\frac{m t}{b m_{s} \bar{\gamma}} \mid \begin{array}{ccc}
-m L & -m-m_{s} & -m L \\
-1 & -m L
\end{array}\right) d t
$$

By using table of integral series and product [19, eq.(7.811.2)] and after some mathematical calculations, we get,

$$
\begin{aligned}
& P_{e}(E)=\frac{\Gamma(0.5)}{2 \pi \Gamma(m L) \Gamma\left(m+m_{s}\right)}\left(\frac{\Gamma\left(m+m_{s}\right)}{\Gamma\left(m_{s}\right)}\right)^{L}\left(\frac{m}{b m_{s} \bar{\gamma}}\right)^{m L+1} \\
& \times G_{4,3}^{1,4}\left(\frac{m}{b m_{s} \bar{\gamma}} \mid \begin{array}{cccc}
-m L-0.5 & -m L & -m-m_{s} & -m L \\
-1 & -m L & -m L-1
\end{array}\right)
\end{aligned}
$$

\section{B. BDPSK and NCFSK}

Average BER for binary differential phase shifting keying (BDPSK) and noncoherent frequency shift keying (NCFSK) using MGF approach can be obtained by [20],

$$
P_{e}(E)=0.5 \cdot M_{M R C}(a)
$$

By substituting (8) into (14), we get,

$$
\begin{aligned}
P_{e}(E) & =\frac{0.5}{\Gamma(m L) \Gamma\left(m+m_{s}\right)}\left(\frac{m}{a m_{s} \bar{\gamma}}\right)^{m L+1}\left(\frac{\Gamma\left(m+m_{s}\right)}{\Gamma\left(m_{s}\right)}\right)^{L} \\
& \times G_{3,2}^{1,3}\left(\frac{m}{a m_{s} \bar{\gamma}} \mid \begin{array}{cc}
-m L & -m-m_{s} \\
-1 & -m L
\end{array}\right)
\end{aligned}
$$

where, $\mathrm{a}=1$ for BDPSK and $\mathrm{a}=0.5$ for NCFSK modulation scheme.

\section{M-ary Quadrature Amplitude Modulation}

Probability of error for square MQAM can be defined as [20],

$$
\begin{aligned}
P_{e}(E) & =\frac{4}{\pi \log _{2}(M)}\left\{\left(1-\frac{1}{\sqrt{M}}\right) \int_{0}^{\pi / 2} M_{M R C}\left(\frac{g_{M Q A M}}{\sin ^{2}(\theta)}\right) d \theta\right. \\
& \left.-\left(1-\frac{1}{\sqrt{M}}\right)^{2} \int_{0}^{\pi / 4} M_{M R C}\left(\frac{g_{M Q A M}}{\sin ^{2}(\theta)}\right) d \theta\right\}
\end{aligned}
$$

where, $g_{M Q A M}=3 \log _{2}(M) / 2(M-1)$

With the help of [21, eq.(19)], we get,

$$
\begin{aligned}
& P_{e}(E)=\frac{4}{\pi \log _{2}(M)}\left[( 1 - \frac { 1 } { \sqrt { M } } ) \left\{\frac{1}{12} M_{M R C}\left(g_{M Q A M}\right)\right.\right. \\
& \left.\left.+\frac{1}{4} M_{M R C}\left(\frac{4}{3} g_{M Q A M}\right)\right\}-\left(1-\frac{1}{\sqrt{M}}\right)^{2}\left\{\frac{1}{8} M_{M R C}\left(2 g_{M Q A M}\right)\right\}\right]
\end{aligned}
$$

By substituting (8) into (17), we get,

$P_{e}(E)=\frac{4}{\pi \log _{2}(M) \Gamma(m L) \Gamma\left(m+m_{s}\right)}\left(\frac{\Gamma\left(m+m_{s}\right)}{\Gamma\left(m_{s}\right)}\right)^{L}\left(\frac{m}{m_{s} \bar{\gamma}}\right)^{m L+1}$

$\left[\left(1-\frac{1}{\sqrt{M}}\right)\left\{\frac{1}{12}\left(\frac{1}{g_{\text {MQAM }}}\right)^{m L+1} G_{3,2}^{1,3}\left(\frac{m}{m_{s} \bar{\gamma} g_{\text {MQAM }}} \mid \begin{array}{ccc}-m L & -m-m_{s} & -m L \\ -1 & -m L\end{array}\right)\right.\right.$

$\left.+\frac{1}{4}\left(\frac{3}{4 g_{\text {MQAM }}}\right)^{m L+1} G_{3,2}^{1,3}\left(\frac{3 m}{4 m_{s} \bar{\gamma} g_{M Q A M}} \mid \begin{array}{ccc}-m L & -m-m_{s} & -m L \\ -1 & -m L\end{array}\right)\right\}$

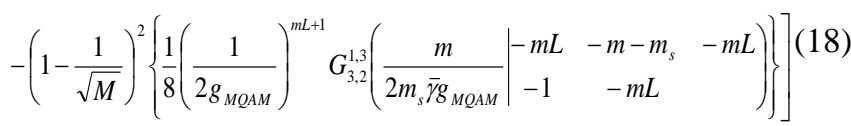


This is the exact closed-form expression of ABER for MQAM modulation scheme over Fisher fading channel.

A tight upper bound expression of conditional BER for MQAM over AWGN channel is given by [22],

$$
P_{e}(E / \gamma) \leq 0.2 \exp \left(\sqrt{\frac{1.5 \gamma}{(M-1)}}\right), \quad \gamma \geq 0, M \geq 4
$$

By substituting (19) into (9), we obtained,

$$
P_{e}(E)=0.2 \int_{0}^{\infty} \exp \left(-\frac{1.5 \gamma}{(M-1)}\right) f_{M R C}(\gamma) d \gamma
$$

By the definition of MGF, as given in (3), (20) can be written as,

$$
P_{e}(E)=0.2 M_{M R C}\left(\frac{1.5}{(M-1)}\right)
$$

On substituting (8) into (21), we get,

$$
\begin{aligned}
& P_{e}(E)=\frac{0.2}{\Gamma(m L) \Gamma\left(m+m_{s}\right)}\left(\frac{m(M-1)}{1.5 m_{s} \bar{\gamma}}\right)^{m L+1} \\
& \times\left(\frac{\Gamma\left(m+m_{s}\right)}{\Gamma\left(m_{s}\right)}\right)^{L} G_{3,2}^{1,3}\left(\frac{m(M-1)}{1.5 m_{s} \bar{\gamma}} \mid \begin{array}{ccc}
-m L & -m-m_{s} & -m L \\
-1 & -m L
\end{array}\right)
\end{aligned}
$$

This is closed form tight upper bound expression for ABER using MQAM over i.i.d MRC Fisher Snedecor composite fading channel.

\section{M-ary amplitude modulation (MAM)}

ABER for MAM is given by [20],

$$
P_{e}(E)=\frac{2(M-1)}{M \pi \log _{2}(M)} \int_{0}^{\pi / 2} M_{M R C}\left(\frac{g_{A M}}{\sin ^{2} \theta}\right) d \theta
$$

where, $g_{A M}=3 \log _{2}(M) /\left(M^{2}-1\right), \mathrm{M}=2,4,6,8$.

By following the similar step from (10)-(13), we get the solution of (23) as,

$$
P_{e}(E)=\frac{(M-1) \Gamma(0.5)}{\pi M \log _{2}(M) \Gamma(m L) \Gamma\left(m+m_{s}\right)}\left(\frac{\Gamma\left(m+m_{s}\right)}{\Gamma\left(m_{s}\right)}\right)^{L}
$$

$\times\left(\frac{m}{g_{A M} m_{s} \bar{\gamma}}\right)^{m L+1} G_{4,3}^{1,4}\left(\frac{m}{g_{A M} m_{s} \bar{\gamma}} \mid \begin{array}{cccc}-m L-0.5 & -m L & -m-m_{s} & -m L \\ -1 & -m L & -m L-1\end{array}\right)$

\section{E. M-ary phase shift keying (MPSK)}

The SER for coherent MPSK is defined by [2],

$$
P_{e}(E)=\frac{1}{\pi} \int_{0}^{\pi-\pi / M} M_{M R C}\left(\frac{g_{M P S K}}{\sin ^{2}(\theta)}\right) d \theta
$$

where, $g_{M Q A M}=\sin ^{2}(\pi / M)$

With the help of [21, eq.16], (25) becomes

$$
\begin{aligned}
P_{e}(E) & =\left(\frac{\Theta}{2 \pi}-\frac{1}{6}\right) M_{M R C}\left(g_{M P S K}\right)+\frac{1}{4} M_{M R C}\left(\frac{4}{3} g_{M P S K}\right) \\
& +\left(\frac{\Theta}{2 \pi}-\frac{1}{4}\right) M_{M R C}\left(\frac{g_{M P S K}}{\sin ^{2} \Theta}\right)
\end{aligned}
$$

where, $\Theta=\pi-\pi / M$

By substituting (8) in (26), we get

$P_{e}(E)=\frac{1}{\Gamma(m L) \Gamma\left(m+m_{s}\right)}\left(\frac{m}{m_{s} \bar{\gamma}}\right)^{m L+1}\left(\frac{\Gamma\left(m+m_{s}\right)}{\Gamma\left(m_{s}\right)}\right)^{L}\left[\left(\frac{\Theta}{2 \pi}-\frac{1}{6}\right)\left(\frac{1}{g_{M P S K}}\right)^{m L+1}\right.$

$$
\begin{aligned}
& \times G_{3,2}^{1,3}\left(\frac{m}{m_{s} \bar{\gamma} g_{M P S K}} \mid \begin{array}{ccc}
-m L & -m-m_{s} & -m L \\
-1 & -m L
\end{array}\right) \\
&+ \frac{1}{4}\left(\frac{3}{4 g_{M P S K}}\right)^{m L+1} G_{3,2}^{1,3}\left(\frac{3 m}{4 m_{s} \bar{\gamma} g_{M P S K}} \mid \begin{array}{ccc}
-m L & -m-m_{s} & -m L \\
-1 & -m L
\end{array}\right) \\
&+\left(\frac{\Theta}{2 \pi}-\frac{1}{4}\right)\left(\frac{\sin ^{2} \Theta}{g_{M P S K}}\right)^{m L+1} G_{3,2}^{1,3}\left(\frac{m \sin ^{2} \Theta}{m_{s} \bar{\gamma} g_{M P S K}} \mid \begin{array}{ccc}
-m L & -m-m_{s} & -m L \\
-1 & -m L
\end{array}\right)
\end{aligned}
$$

\section{F. Noncoherent M-ary Frequency shift keying (NCMFSK)}

The conditional SER for NCMSFK over the AWGN channel is given by [23],

$$
P_{e}(E / \gamma)=\frac{1}{M} \sum_{i=2}^{M}(-1)^{i}\left(\begin{array}{c}
M \\
i
\end{array}\right) \exp \left(-\left(1-\frac{1}{i}\right) \gamma\right)
$$

By plugging (28) into (9), we get the ASER for NCMFSK modulation scheme as,

$$
P_{e}(E)=\frac{1}{M} \sum_{i=2}^{M}(-1)^{i}\left(\begin{array}{c}
M \\
i
\end{array}\right) \int_{0}^{\infty} \exp \left(-\left(1-\frac{1}{i}\right) \gamma\right) f_{\gamma}(\gamma) d \gamma
$$

The integration term in (29) is the definition of MGF, as given in (3), therefore (29) becomes,

$$
P_{e}(E)=\frac{1}{M} \sum_{i=2}^{M}(-1)^{i}\left(\begin{array}{c}
M \\
i
\end{array}\right) M_{M R C}\left(\left(1-\frac{1}{i}\right)\right)
$$

Now, by substituting (8) into (30), we obtained,

$$
\begin{aligned}
& P_{e}(E)=\frac{1}{M} \sum_{i=2}^{M}(-1)^{i}\left(\begin{array}{c}
M \\
i
\end{array}\right) \frac{1}{\Gamma(m L) \Gamma\left(m+m_{s}\right)}\left(\frac{m}{\left(1-\frac{1}{i}\right) m_{s} \bar{\gamma}}\right)^{m L+1} \\
& \times\left(\frac{\Gamma\left(m+m_{s}\right)}{\Gamma\left(m_{s}\right)}\right)^{L} G_{3,2}^{1,3}\left(\frac{m}{\left(1-\frac{1}{i}\right) m_{s} \bar{\gamma}} \mid \begin{array}{ccc}
-m L & -m-m_{s} & -m L \\
-1 & -m L
\end{array}\right)
\end{aligned}
$$

\section{CHANNEL CAPACITY ANALYSIS}

The expressions of channel capacity for ORA and CIFR policy will be derived in this section. These two capacities will be obtained by using newly derived MGF expression

\section{A. Optimal rate adaptation with constant transmit power (ORA)}

In this policy, the transmitter has constant transmit power and receiver has knowledge the channel state information (CSI). The channel capacity in bit/sec/Hz under ORA policy is defined as [6],

$$
C_{\text {ORA }}=\frac{1}{\ln (2)} \int_{0}^{\infty} E_{i}(-\omega) M_{M R C}^{\prime}(\omega) d \omega
$$

Where $M_{M R C}^{\prime}(\omega)$ is the first order derivative of MGF $M_{M R C}(\omega)$ with respect to $\omega$, and $\mathrm{E}_{\mathrm{i}}(\cdot)$ represents Exponential integral function [19]. To solve the above integration, first of all, we have to evaluate the first derivative of MGF which can be given as, 


$$
M_{M R C}^{\prime}(\omega)=\frac{d}{d \omega} M_{M R C}(\omega)
$$

By substituting (8) into (32), we obtained,

$$
\begin{aligned}
& M_{M R C}^{\prime}(\omega)=\frac{1}{\Gamma(m L) \Gamma\left(m+m_{s}\right)}\left(\frac{\Gamma\left(m+m_{s}\right)}{\Gamma\left(m_{s}\right)}\right)^{L} \\
& \times \frac{d}{d \omega}\left(\frac{m}{\omega m_{s} \bar{\gamma}}\right)^{m L+1} G_{3,2}^{1,3}\left(\frac{m}{\omega m_{s} \bar{\gamma}} \mid \begin{array}{ccc}
-m L & -m-m_{s} & -m L \\
-1 & -m L
\end{array}\right)
\end{aligned}
$$

With the help of integral series and product, vol.3 [24, eq. (8.2.2.30)] and after some mathematical steps, we get,

$$
\begin{aligned}
& M_{M R C}^{\prime}(\omega)=\frac{-1}{\Gamma(m L) \Gamma\left(m+m_{s}\right)}\left(\frac{\Gamma\left(m+m_{s}\right)}{\Gamma\left(m_{s}\right)}\right)^{L}\left(\frac{m}{m_{s} \bar{\gamma}}\right)^{m L+1} \\
& \times \omega^{-m L-2} G_{3,2}^{1,3}\left(\frac{m}{\omega m_{s} \bar{\gamma}} \mid \begin{array}{ccc}
-m L-1 & -m-m_{s} & -m L \\
-1 & -m L
\end{array}\right)
\end{aligned}
$$

On substituting (35) into (32), we obtained,

$$
\begin{gathered}
C_{O R A}=\frac{-1}{\ln (2) \Gamma(m L) \Gamma\left(m+m_{s}\right)}\left(\frac{\Gamma\left(m+m_{s}\right)}{\Gamma\left(m_{s}\right)}\right)^{L}\left(\frac{m}{m_{s} \bar{\gamma}}\right)^{m L+1} \\
\times \int_{0}^{\infty} E_{i}(-\omega) \omega^{-m L-2} G_{3,2}^{1,3}\left(\frac{m}{s m_{s} \bar{\gamma}} \mid \begin{array}{ccc}
-m L-1 & -m-m_{s} & -m L \\
-1 & -m L
\end{array}\right) d \omega
\end{gathered}
$$

With the help of [24, eq.(8.4.11.1)], (36) can be written as,

$$
\begin{gathered}
C_{O R A}=\frac{1}{\ln (2) \Gamma(m L) \Gamma\left(m+m_{s}\right)}\left(\frac{\Gamma\left(m+m_{s}\right)}{\Gamma\left(m_{s}\right)}\right)^{L}\left(\frac{m}{m_{s} \bar{\gamma}}\right)^{m L+1} \\
\times \int_{0}^{\infty} \omega^{-m L-2} G_{1,2}^{2,0}\left(\omega \mid \begin{array}{ll}
1 & \\
0 & 0
\end{array}\right) G_{3,2}^{1,3}\left(\frac{m}{s m_{s} \bar{\gamma}} \mid \begin{array}{ccc}
-m L-1 & -m-m_{s} & -m L \\
-1 & -m L
\end{array}\right) d \omega
\end{gathered}
$$

By using integral series and product [24, eq. (2.24.1.1)] and property of Meijer $G$ function. After some mathematical calculation, we get the solution of (37) as,

$$
\begin{gathered}
C_{\text {ORA }}=\frac{1}{\ln (2) \Gamma(m L) \Gamma\left(m+m_{s}\right)}\left(\frac{\Gamma\left(m+m_{s}\right)}{\Gamma\left(m_{s}\right)}\right)^{L}\left(\frac{m}{m_{s} \bar{\gamma}}\right)^{m L+1} \\
\times G_{4,4}^{3,3}\left(\begin{array}{cccc}
\frac{m_{s} \bar{\gamma}}{m} & 2+m L & 2+m L & 1+m L \\
2+m L & 1+m+m_{s} & 1+m L & 1+m L
\end{array}\right)
\end{gathered}
$$

This is an exact closed-form expression of channel capacity under ORA transmission protocol.

\section{B. Channel Inversion with Fixed Rate (CIFR)}

In this policy, the transmitter adapts its power to maintain received SNR constant. Channel capacity (b/s/Hz) for CIFR policy using MGF method is given by [17],

$$
C_{C I F R}=\log _{2}(1+\underbrace{\frac{1}{\int_{0}^{\infty} M_{M R C}(\omega) d \omega}}_{P})
$$

For obtaining the channel capacity for CIFR, we have to evaluate the integral term presented in (39) (as denoted by $\mathrm{P}$ ). With the help of (8), P can be written as,

$$
\begin{aligned}
& P=\int_{0}^{\infty} M_{\gamma}(\omega) d \omega=\frac{1}{\Gamma(m L) \Gamma\left(m+m_{s}\right)}\left(\frac{\Gamma\left(m+m_{s}\right)}{\Gamma\left(m_{s}\right)}\right)^{L} \\
& \times \int_{0}^{\infty}\left(\frac{m}{\omega m_{s} \bar{\gamma}}\right)^{m L+1} G_{3,2}^{1,3}\left(\frac{m}{\omega m_{s} \bar{\gamma}} \mid \begin{array}{ccc}
-m L & -m-m_{s} & -m L \\
-1 & -m L
\end{array}\right) d \omega
\end{aligned}
$$

Let $1 / \omega=t$ or $\left(1 / \omega^{2}\right) d \omega=-d t$ and after some mathematical steps, (40) can be written as,

$$
\begin{aligned}
& P=\frac{1}{\Gamma(m L) \Gamma\left(m+m_{s}\right)}\left(\frac{\Gamma\left(m+m_{s}\right)}{\Gamma\left(m_{s}\right)}\right)^{L}\left(\frac{m}{m_{s} \bar{\gamma}}\right)^{m L+1} \\
& \times \int_{0}^{\infty} t^{m L-1} G_{3,2}^{1,3}\left(\frac{m t}{m_{s} \bar{\gamma}} \mid \begin{array}{ccc}
-m L & -m-m_{s} & -m L \\
-1 & -m L
\end{array}\right) d t
\end{aligned}
$$

With the help of the table of integral series and products [12, eq. (7.811.4)] and after some mathematical steps, we obtained

$$
P=\frac{\Gamma\left(1+m+m_{s}-m L\right)}{m L \Gamma\left(m+m_{s}\right)}\left(\frac{\Gamma\left(m+m_{s}\right)}{\Gamma\left(m_{s}\right)}\right)^{L}\left(\frac{m}{m_{s} \bar{\gamma}}\right)
$$

By plugging (42) into (39), we get,

$$
C_{C I F R}=\log _{2}\left(1+\frac{m L \Gamma\left(m+m_{s}\right)}{\Gamma\left(1+m+m_{s}-m L\right)}\left(\frac{\Gamma\left(m_{s}\right)}{\Gamma\left(m+m_{s}\right)}\right)^{L}\left(\frac{m_{s} \bar{\gamma}}{m}\right)\right)(43)
$$

This is the MGF based closed-form expression of channel capacity for CIFR policy under i.i.d Fisher Snedecor composite fading channel using MRC diversity.

\section{NUMERICAL RESULTS AND DISCUSSION}

In this section, the numerical analysis of $\mathrm{L}$ branch independent identically distributed MRC receiver over Fisher fading channel is presented. We plot the numerical results of ABER using different modulation schemes and channel capacity under various adaptive transmission protocol for different fading severity parameter (m) and shadowing parameter $\left(m_{s}\right)$. The MRC diversity technique provides better performance improvement as compared to SISO system.

Fig. 1 shows the ABER for BPSK modulation scheme as the function of average SNR (dB) of Fisher fading model with single branch MRC receiver $(\mathrm{L}=1)$. We have compared the result of ABER for BPSK modulation scheme over Fisher fading channel (solid line) with ABER of Rayleigh (Asterisk) and Nakagami-m distribution (circle) over single branch receiver $(L=1)$. The result shows that, with $m=1, m_{s}=20$ and $\mathrm{L}=1$, the BER for BPSK ((13) with $\mathrm{b}=1)$ overlapped with BER of Rayleigh distribution [1, eq. 4.134]. Similarly, for $\mathrm{m}=2, \mathrm{~m}_{\mathrm{s}}=20$ and $\mathrm{L}=1$, (13) coincides with the BER of Nakagami-m distribution [1, eq. 4.138]. Fig.1 shows that, on increasing the fading severity parameter $(\mathrm{m}=1$ to 3 ) with constant shadowing parameter $\left(\mathrm{m}_{\mathrm{s}}=20\right)$ and $\mathrm{L}=1$, the BER decreases.

Fig. 2 represents the plot of ABER for BPSK, BFSK with minimum correlation and BFSK modulation schemes over one, two and three branch MRC receiver with constant fading severity $(\mathrm{m}=1)$ and shadowing parameter $\left(\mathrm{m}_{\mathrm{s}}=2\right)$. From Fig.2, it is observed that on increasing the number of branches ( $\mathrm{L}=1$ to 3 ) for BPSK or BFSK modulation scheme, the BER decreases that improve the system performance. Fig. 2 also depicts that, the BPSK modulation scheme has lower BER as compared to BFSK modulation scheme for any value of $\mathrm{L}$.

Fig. 3 depicts the plot of ABER for BDPSK and NCFSK modulation scheme over one, two and three branch MRC receiver with $\mathrm{m}=1$ and $\mathrm{m}_{\mathrm{s}}=20$. The study shows that on increasing the number of branches (L) at the receiver section, the BER performance increases for BDSPK (or NCFSK) 
modulation schemes as shown in Fig. 3. Also, Fig.3 shows that the BER for BDPSK modulation scheme is lower than NCFSK modulation for any value of $\mathrm{L}$. Moreover, for $\mathrm{m}=1$ under light shadowing $\left(\mathrm{m}_{\mathrm{s}}=20\right)$ with $\mathrm{L}=1$, the BER using BDPSK (or NCFSK) modulation scheme of Fisher fading model (solid line) coincides with BER of Rayleigh distribution [2].

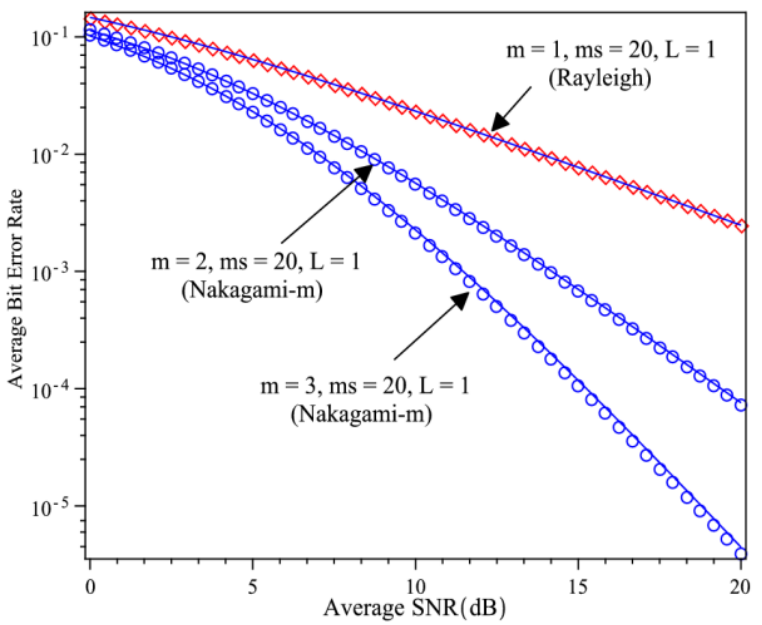

Fig. 1 ABER for BPSK modulation schemes versus average SNR over single branch MRC receiver with $\mathrm{m}=1,2,3$ and $\mathrm{m}_{\mathrm{s}}$ $=20$.

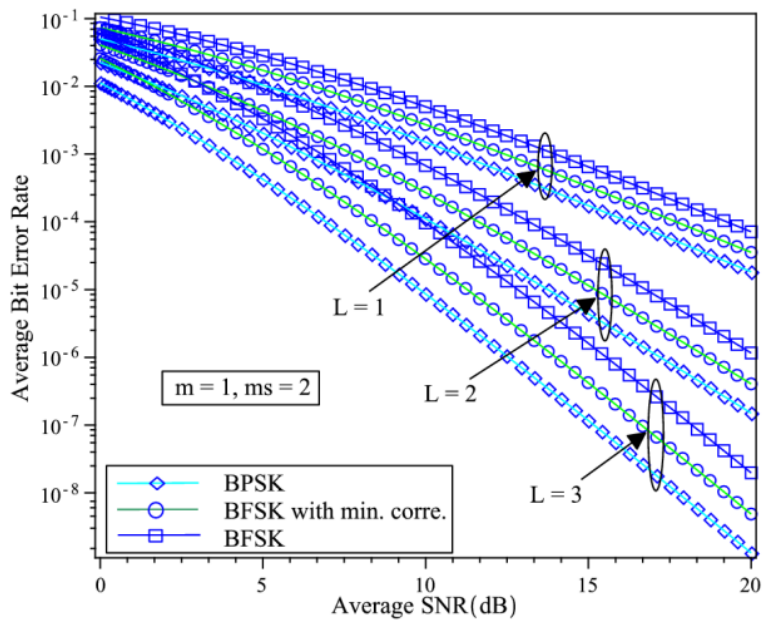

Fig. 2 ABER for BPSK, BFSK and BFSK with minimum correlation for $\mathrm{m}=1, \mathrm{~m}_{\mathrm{s}}=2$ and $\mathrm{L}=1,2,3$.

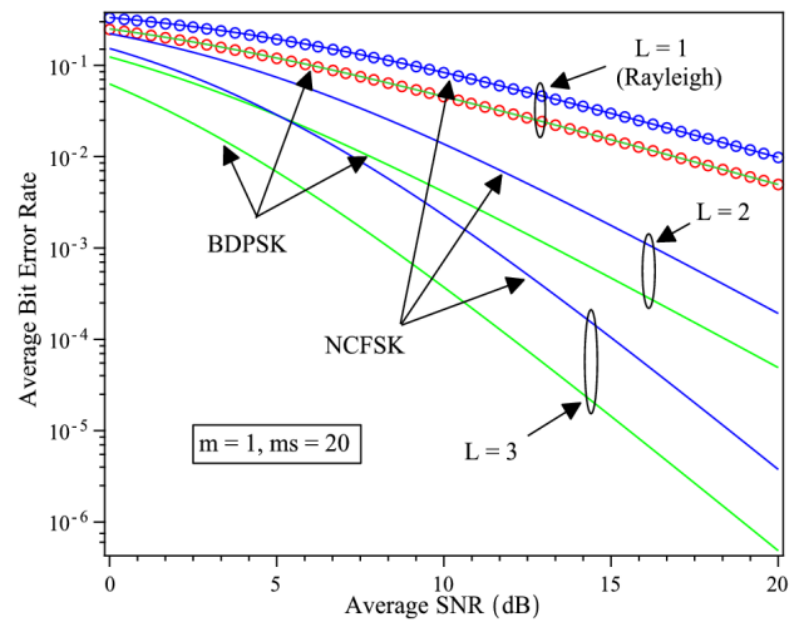

Fig. 3 ABER for BDPSK and NCFSK modulation schemes versus average $\mathrm{SNR}$ with $\mathrm{MRC}(\mathrm{L}=1,2,3)$. $\mathrm{F}$ fading model (solid line), Rayleigh (circle).

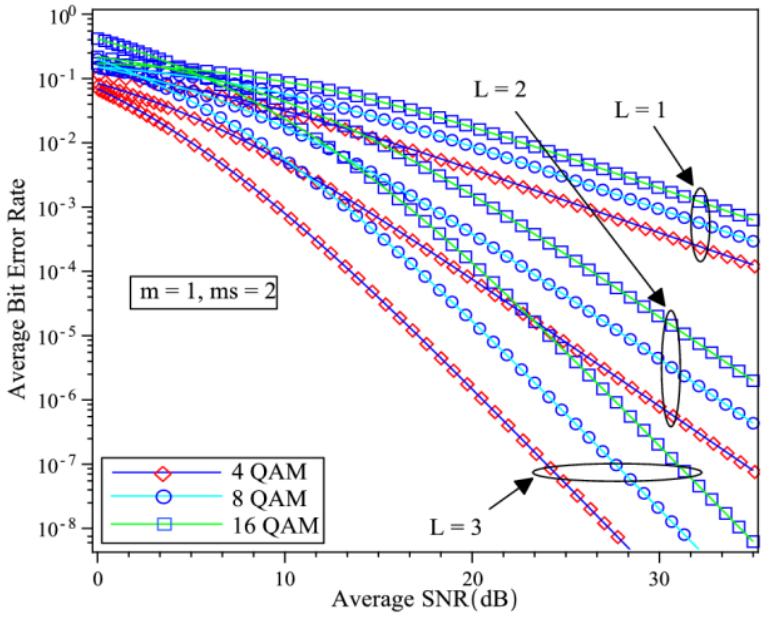

Fig. 4 ABER for MQAM modulation schemes versus average SNR with MRC $(\mathrm{L}=1,2,3)$.

Fig.4 represents the plot of ABER for 4 QAM, 8QAM and 16 QAM modulation schemes (18) with MRC reception algorithm. We have used $\mathrm{m}=1, \mathrm{~m}_{\mathrm{s}}=2$ and $\mathrm{L}=1,2,3$ to plot the error rate curve. From Fig. 4, it is observed that, on increasing the value of $\mathrm{M}$ ( $\mathrm{M}=4$ to 16$)$ for fixed $\mathrm{L}$ (1, 2 or 3 ), the BER increases that degrade the system performance. Also, on increasing the number of branches ( $L=1$ to 3 ) with constant M (4, 8 or 16$)$, the BER decreases that improve the system performance.

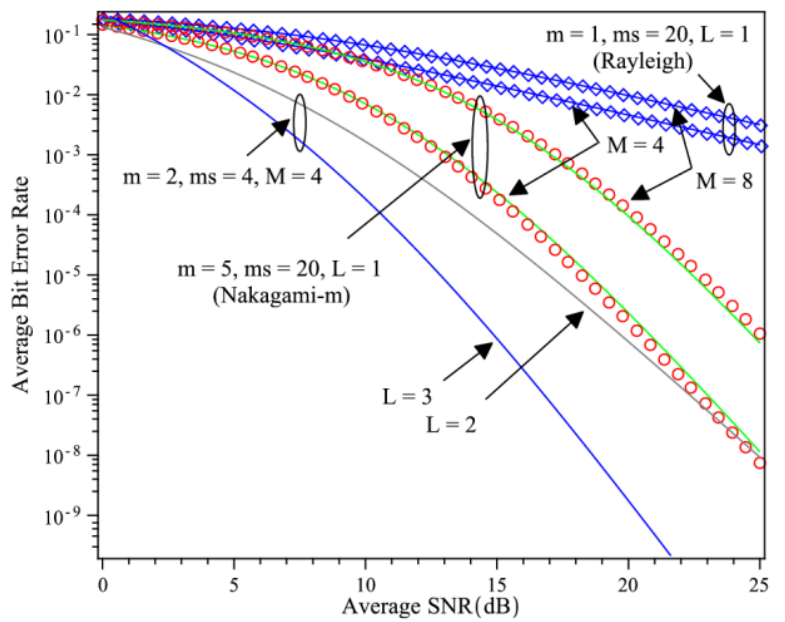

Fig.5 ABER using $4 \mathrm{AM}$ and 8 AM modulation schemes versus average $\mathrm{SNR}$ for different value of $\mathrm{m}$ and $\mathrm{m}_{\mathrm{s}}$ with MRC ( $\mathrm{L}=1,2,3)$. F fading (solid line), Rayleigh (diamond), Nakagami-m (circle).

Fig.5 represents the ABER for MAM modulation scheme over $\mathrm{F}$ fading channel (solid line) compared with Rayleigh (diamond) and Nakagami-m distribution (circle). From Fig. 5, it is observed that, for $\mathrm{m}=1$ under light shadowing $\left(\mathrm{m}_{\mathrm{s}}=20\right)$ with $L=1$ and $M=4$ (or 8), the BER for MAM (24) coincides with BER of Rayleigh distribution [2, eq.8.103]. In a similar way, with $\mathrm{m}=5$ under light shadowing $\left(\mathrm{m}_{\mathrm{s}}=20\right)$ with $\mathrm{L}=1$ and $\mathrm{M}=4$ (or 8), (24) reduces to BER of Nakagami-m distribution [2, eq.8.106]. Fig. 5 also illustrates that, on increasing the number of branches $(\mathrm{L}=2$ to 3$)$ with $\mathrm{m}=2$,

Published By: 
$\mathrm{m}_{\mathrm{s}}=4$ and $\mathrm{M}=4$, the error rate performance improves.

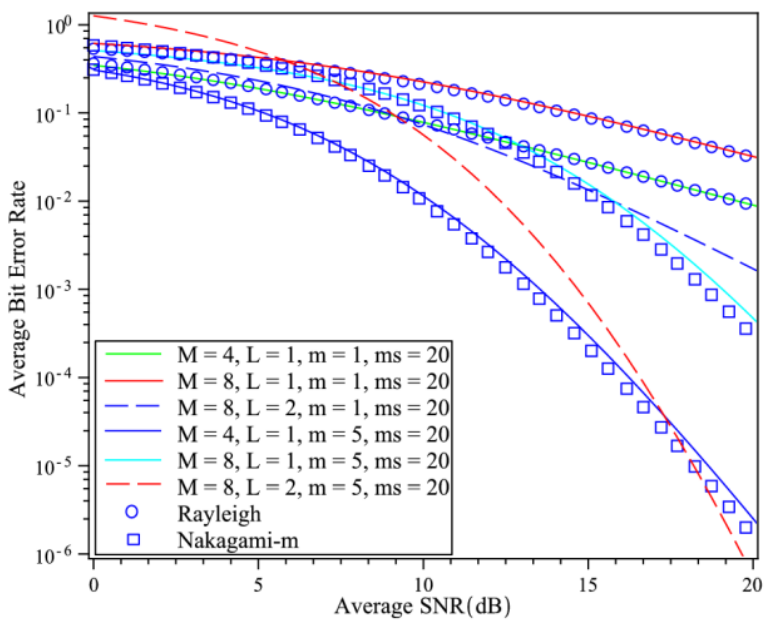

Fig. 6 ABER for MPSK modulation schemes $(M=4,8)$ versus average $\mathrm{SNR}$ for $\mathrm{m}=1,5$ and $\mathrm{m}_{\mathrm{s}}=20$ with $\mathrm{MRC}(\mathrm{L}=$ 1, 2). F fading (solid line), Rayleigh (circle), Nakagami-m (square).

Fig.6 shows the plot of ABER for 4 PSK and 8 PSK modulation scheme (27) over single and dual branch MRC reception with $\mathrm{m}=1,5$ and $\mathrm{m}_{\mathrm{s}}=20$. The proposed BER results reduce to previously reported results of other fading distribution such as Rayleigh and Nakagami-m distribution which is the special case of Fisher fading model. The study shows that for $\mathrm{m}=1$ under light shadowing $\left(\mathrm{m}_{\mathrm{s}}=20\right)$ with $\mathrm{M}$ $=4$ (or 8) and $\mathrm{L}=1$, the ABER for MPSK modulation scheme reduces to ABER of Rayleigh distribution [2, eq.8.113]. Likewise, for $\mathrm{m}=5$ under light shadowing $\left(\mathrm{m}_{\mathrm{s}}=20\right)$ with $\mathrm{M}=$ 4 (or 8), (27) overlapped with BER of Nakagami-m distribution [2, eq.8.115]. Fig.6 also represent that, on increasing the value of $\mathrm{M}$ (4 to 8 ) with the constant value of $\mathrm{L}, \mathrm{m}$ and $\mathrm{m}_{\mathrm{s}}$, the error rate performance decreases. Also, on increasing the number of branches ( $\mathrm{L}=1$ to 2 ) while $\mathrm{m}, \mathrm{m}_{\mathrm{s}}$ and $\mathrm{M}$ kept constant, the ABER decreases that improve the system performance.

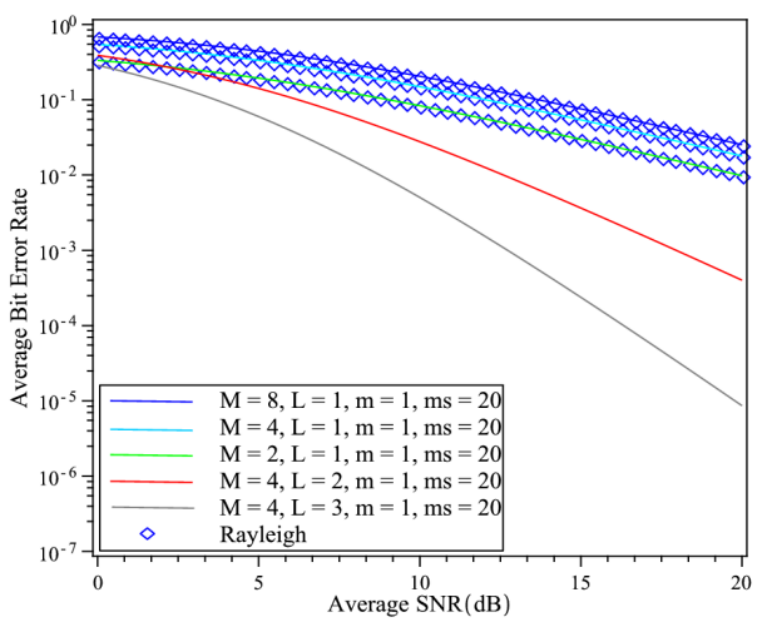

Fig. 7 ABER for NCMFSK modulation schemes $(M=2,4,8)$ versus average $S N R$ for $m=1$ and $m_{s}=20$ with $M R C(L=1$, 2). F fading (solid line), Rayleigh (square).

Fig. 7 illustrate the plot of ABER for noncoherent MFSK modulation scheme (31) over one, two and three branch MRC receiver with $\mathrm{M}=2,4,8, \mathrm{~m}=1$ and $\mathrm{m}_{\mathrm{s}}=20$. From fig. 7 , it is observed that, on increasing the values of $\mathrm{M}$ (2 to 8 ) with $\mathrm{L}=$ $1, \mathrm{~m}=1$ and $\mathrm{m}_{\mathrm{s}}=20$, the BER increases. Also, for $\mathrm{m}=1$ and $\mathrm{m}_{\mathrm{s}}=20$ over single branch with $\mathrm{M}=2$ (or 4 or 8 ), the BER for NCMFSK modulation reduces to BER of Rayleigh distribution [23, eq.9]. Moreover, on increasing the number of branches ( $\mathrm{L}=1$ to 3 ) with $\mathrm{m}=1, \mathrm{~m}_{\mathrm{s}}=20$ and $\mathrm{M}=4$, the error rate performance increases, as shown in fig.7.

Fig. 8 shows the plot of average channel capacity (in $\mathrm{bit} / \mathrm{sec} / \mathrm{Hz}$ ) under ORA transmission protocol with $\mathrm{m}=1,2.5$, $\mathrm{m}_{\mathrm{s}}=20$ and $\mathrm{L}=1,2,3$. We have compared the numerical results derived in (38) with channel capacity of Rayleigh (circle) and Nakagami-m distribution (square). For, $\mathrm{m}=1, \mathrm{~m}_{\mathrm{s}}$ $=20$ over single branch, channel capacity of Fisher fading model (38) coincides with channel capacity of Rayleigh distribution [1, eq.4.208]. Also, for $\mathrm{m}=2.5, \mathrm{~m}_{\mathrm{s}}=20$ and $\mathrm{L}=$ 1, (38) agreed with channel capacity of Nakagami- $m$ distribution [1, eq.4.207]. Fig. 8 also shows that on increasing the number of branches ( $\mathrm{L}=1$ to 3 ) with $\mathrm{m}=1$ under light shadowing $\left(\mathrm{m}_{\mathrm{s}}=20\right)$, the channel capacity improves.

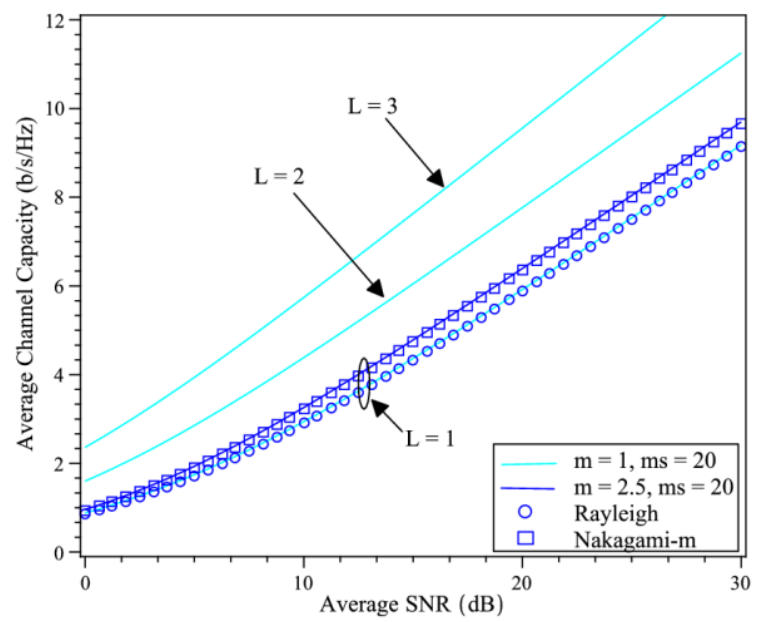

Fig. 8 Channel capacity under ORA policy versus average SNR $(\mathrm{dB})$ for $\mathrm{m}=1,2.5, \mathrm{~m}_{\mathrm{s}}=20$ and $\mathrm{L}=1,2,3$. F Fading (solid line), Rayleigh (circle), Nakagami-m (square).

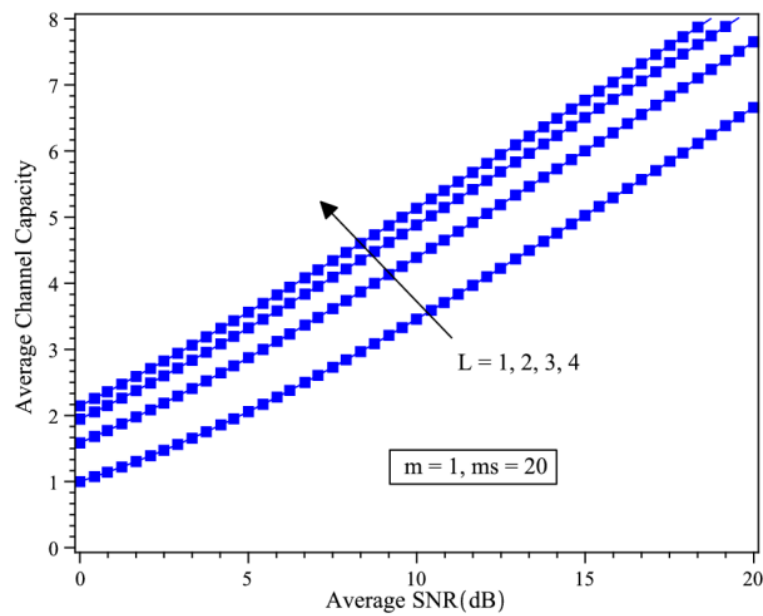

Fig. 9 Channel capacity under CIFR policy versus average SNR for $\mathrm{m}=1, \mathrm{~m}_{\mathrm{s}}=20$ and $\mathrm{L}=1,2,3,4$.

Fig. 9 illustrates the plot of average channel capacity (bit/sec/Hz) over CIFR transmission protocol with $\mathrm{m}$ $=1, \mathrm{~m}_{\mathrm{s}}=20$ and $\mathrm{L}=1,2,3$, 4. From Fig. 9, it is observed

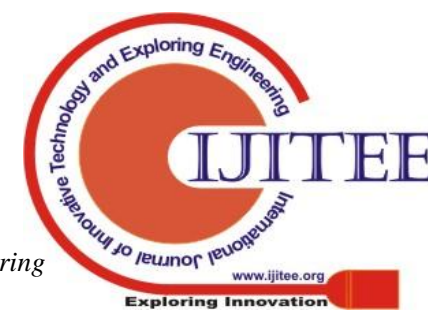


that, on increasing the number of branches $(\mathrm{L}=1$ to 4$)$ with $\mathrm{m}$ $=1$ under light shadowing $\left(\mathrm{m}_{\mathrm{s}}=20\right)$, the channel capacity improves.

\section{CONCLUSIONS}

The performance evaluation of Fisher Snedecor composite model with $\mathrm{L}$ branch MRC receiver has been presented in this paper. In this paper, the expression for MGF of instantaneous SNR using independent identically distributed (i.i.d) MRC diversity technique has been derived. By using newly derived MGF, the expressions of ABER for BPSK, BFSK, BDPSK, NCFSK, MQAM, MAM, MPSK and NCMFSK modulation schemes have been derived. Further, the expressions of ACC for ORA and CIFR policy have been obtained. All the derived expressions are presented in the closed form. The most commonly available simulation software such as MATLAB and MAPLE has been used to implement the numerical results of derived expressions. The obtained numerical results show good agreement with the results of Rayleigh and Nakagami-m distribution that are special case of Fisher Snedecor composite fading model. The performance improvements have been seen by increasing the number of branches (L) at the receiver section.

\section{REFERENCES}

1. P.M. Shankar, Fading and Shadowing for Wireless system. New York: Springer, 2005.

2. M.K. Simon, M.S. Alouini, Digital Communication over Fading Channels. New York: John Wiley \& Sons, 2005.

3. M.K. Simon, M.S. Alouini, A unified approach to the performance analysis of digital communication over generalized fading channels. Proceedings of the IEEE, 1999; 86(9): 1860-1877.

4. M.S. Alouini, A. J. Goldsmith. (1999). A unified approach for calculating error rates of linearly modulated signals over generalized fading channels. IEEE Trans. commun. 47(9), pp.1324-1334.

5. K.A. Hamdi. (2008). Capacity of MRC on correlated Rician fading channels. IEEE Trans. commun. 56(5), pp. 708-711.

6. M.D. Renzo, F. Graziosi, F. Santucci. (2010). Channel capacity over generalized fading channels: A novel MGF-based approach for performance analysis and design of wireless communication systems. IEEE Trans. Veh. Technol. 59(1), pp. 127-149.

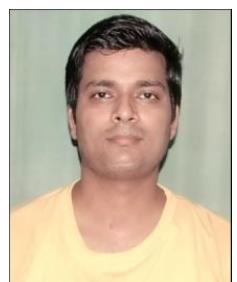

7. A. Abdi, M. Kaveh. (1998). K-distribution: an appropriate substitute for Rayleigh-lognormal distribution in fading- shadowing wireless channels. Electron. Lett. 34(9), pp. 851-852.

8. P.M. Shankar. (2004). Error rates in generalized shadowed fading channels. Wirel. Pers. Commun. 3, pp. 233-38.

9. S.K. Yoo, S.L. Cotton, P.C. Sofotasios, S. Freear. (2016). Shadowed Fading in Indoor Off-Body Communication Channels: A Statistical Characterization Using the $\kappa-\mu /$ gamma Composite Fading Model. IEEE Trans. Wirel. Commun. 15(8), pp. 5231-5244.

10. S.K. Yoo, S.L. Cotton, P.C. Sofotasios, M. Matthaiou, M. Valkama, G.K. Karagiannidis. (2017). The Fisher-Snedecor F distribution: A Simple and Accurate Composite Fading Model. IEEE Commun Lett. 21(7), pp. 1661-1664.

11. T. Aldalgamouni, M.C. Ilter, O.S. Badarneh, H. Yanikomeroglu. Performance analysis of Fisher-Snedecor F composite fading channels. IEEE Middle East and North Africa Communications Conference (MENACOMM). Jounieh, Lebanon, 2018.

12. H.A. Hmood, Performance of cognitive radio systems over $\kappa-\mu$ shadowed with integer $\mu$ and Fisher-Snedecor $F$ fading channels. International Iraqi Conference on Engineering Technology and its Applications. Al-Najaf, Iraq, 2018.

13. J. Gong, H. Lee, J. Kang. (2018). Generalized moment generating function-based secrecy performance analysis over Fisher-Snedecor composite fading channels. Electron. Lett. 54 (24), pp. 1381-1383.
14. S. Chen, J. Zhang, G.K. Karagiannidis, B. Ai. (2018). Effective rate of MISO systems over Fisher-Snedecor $\mathrm{F}$ Fading Channels. IEEE Commun. Lett. 22 (12), pp. 2619-2622.

15. L. Kong, G. Kaddoum. (2018). On physical layer security over the Fisher- Snedecor F wiretap Fading Channels. IEEE Access. 6, pp. 39466-39472.

16. F.S. Almehmadi, O.S. Badarneh. (2018). On the effective capacity of Fisher-Snedecor F fading channels. Electon. Lett. 54(18), pp. 1068-1070.

17. S.K. Yoo, P. C. Sofotasios, S.L Cotton, S. Muhaidat, F.J. Lopez-Martinez, J.M. Romero-Jerez, G.K. Karagiannidis. (2019). A comprehensive analysis of the achievable channel capacity in $\mathrm{F}$ composite fading channels. IEEE Access. 2019.

18. O.S. Badarneh, D.B. da Costa, P. C. Sofotasios, S. Muhaidat, S.L. Cotton. (2018). On the sum of Fisher-Snedecor F variates and its application to maximal-ratio combining. IEEE Wirel. Commun. Lett. 7(6), pp. 966-969.

19. I.S. Gradshteyn, I.M. Ryzhik. Table of Integrals, Series, and Products. Academic Press, New York, 2007.

20. K.P. Peppas, H.E. Nistazakis, G.S. Tombras. An overview of the physical insight and the various performance metrics of fading channels in wireless communication systems. Advanced Trends Wirel. Commun. In Tech. 2011.

21. S.P. Singh, S. Kumar. (2016). A MGF based closed form expressions for error probability and capacity over EGK fading for interference limited system. Wirel pers commun. 91(2), pp. 577-816.

22. A. Hamed, M. Alsharef, R.K. Rao. MGF based performance analysis of digital wireless system in urban shadowing environment. Proceedings of the world congress on engineering and computer science (WCECS), San Francisco, USA, 2015

23. J. Sun, I.S. Reed. (1999). Performance of MDPSK, MPSK, and noncoherent MFSK in wireless Rician fading channels. IEEE Trans. commun. 47(6), pp. 813-816.

24. Y.A. Brychkov, O.I. Marichev, A.P. Prudnikov. Integrals and Series, vol 3: more special functions. Gordon and Breach science publishers; 1986.

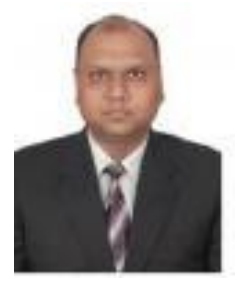

\section{AUTHORS PROFILE}

Hari Shankar received B. Tech and M. Tech degree in Electronics and Communication Engineering from U.P Technical University, Uttar Pradesh, India. He is currently pursuing Ph.D degree in Electronic and Communication Engineering Department at Thapar Institute of Engineering and Technology, Patiala, Punjab, India. His research interest includes wireless communication, indoor channel modeling and wearable communication.

Ankush Kansal received his B. Tech. and M. Tech. Degree in Electronics and Communication Engineering from PTU, Jalandhar and Ph. D. from Thapar Institute of Engineering and Technology, Patiala in the area of Wireless Communication. He is currently working as Assistant Professor in Thapar Institute of Engineering and Technology, Patiala. He has published 25 research articles in referred international journals, international conference and national conference. He is a life time member of ISTE. His research interest includes Networking, Wireless Communication, Image Processing and Embedded Systems. 